\title{
An Iterative Power Allocation Scheme for Spread Spectrum Wireless Systems
}

\author{
Caimu Tang \\ Tut Systems \\ Lake Oswego, OR 97035 \\ ctang@tutsys.com
}

\author{
Anthony T. Chronopoulos \\ Dept. of Computer Science \\ Univ. of Texas at San Antonio \\ San Antonio, TX 78249 \\ atc@cs.utsa.edu
}

\author{
Paul Cotae \\ Dept. of Elec. Engneering \\ Univ. of Texas at San Antonio \\ San Antonio, TX 78249 \\ Paul.Cotae@utsa.edu
}

\begin{abstract}
This paper formulates a new optimization problem for direct-sequence spread spectrum systems by considering the joint transmitted power and link quality in a functional form. Link quality is measured in terms of signal interference to noise ratio including the cross correlations and multipath effect. By solving this optimization problem a new distributed power control scheme is proposed which guarantees a global optimum in all practical cases. The implementation of the proposed procedure can be simply performed locally and the scheme fits well into low-power radios.
\end{abstract}

\section{INTRODUCTION}

In direct-sequence spread spectrum (DSSS) systems, radio channel is normally interference limited. DSSS systems mitigate interference by assigning orthogonal or low crosscorrelated spreading codes to communicating pairs. Here, we point out that out-of-phase autocorrelation (i.e. correlation sidelobe) could seriously degrade the link quality in DSSS networks. Previous work [6], [7] on power allocation has not taken this cross-interference including multipath components and self-interference into account. The decision statistic $Z$ on received signal strength for a link can be decomposed into four components as follows: $Z=I+I_{m}+\zeta+\sigma^{2}$, where $I$ is the desired contribution from the transmitter; $I_{m}$ is the undesired contribution from the transmitter due to multi-path; $\zeta$ is the interference aggregate from $N$ co-channel users caused by cross-correlation, and $\sigma^{2}$ is the undesired contribution from additive white Gaussian noise (AWGN).

We first introduce notations and assumptions to be used throughout the paper.

\section{Notation:}

1) All the vectors will be in bold face letters.

2) $N=$ the number of simultaneous co-channel users or simultaneous transmission pairs or the total number of simultaneous active radio links.

3) We define a vector denoted by $\mathbf{s} \otimes \mathbf{p}$ derived from two

\footnotetext{
${ }^{1}$ The work of Dr. Anthony T. Chronopoulos was supported by NSF grant CCR-0312323. Dr. Tang was at University of Southern California, when this research was performed.
}

vectors

$$
\begin{aligned}
\mathbf{s} & =\left(s_{1}, s_{2}, \cdots, s_{N}\right)^{t r} \\
\mathbf{p} & =\left(p_{1}, p_{2}, \cdots, p_{N}\right)^{t r} \\
\mathbf{s} \otimes \mathbf{p} & =\left(s_{1} p_{1}, s_{2} p_{2}, \cdots, s_{N} p_{N}\right)^{t r}
\end{aligned}
$$

where, $t r$ is the transpose operator.

4) The power vector: $\mathbf{p}=\left(p_{1}, p_{2}, \cdots, p_{N}\right)^{t r}$ is a vector with the $\mathrm{i}$-th radio uses the power $p_{i}$.

5) Let $<x, y>_{N, k}$ be the inner product of two $\mathrm{N}$ dimensional vectors without the $\mathrm{k}$-th term.

6) Denote $K$ the number of multi-paths and the variance $\sigma^{2}$ of the AWGN.

7) $\tilde{\lambda}_{0} \geq 0$ is a properly chosen scalar constant.

8) $\lambda$ is an $\mathrm{N}$ dimension vector and its $\mathrm{i}$-th element is $\lambda_{i}>$ 0 .

9) $\mathrm{SINR}_{i}$ is the signal interference-noise ratio (SINR) of the i-th link.

10) The transmitter/receiver node is denoted by Tx/Rx respectively.

11) Channel in this paper is assumed to be a quasi-state fading channel; futhermore, channel state information in terms of path gains are available to the transmitters.

12) $T$ is the period in number of chip cycles of spread codes.

13) $g_{k k}$ is the path gain between the k-th transmitter and the $\mathrm{k}$-th receiver which accounts for terrestrial attenuation or the free-space propagation for the case where antenna altitudes are sufficient high.

14) $\mathbf{G}_{\mathbf{k}}=\left(g_{1 k}, g_{2 k}, \cdots, g_{N k}\right)^{t r}$.

Since our scheme is applicable to packet radio networks [13], channel state information can easily be made available to transmitters, e.g. piggyback on transmission from neighbors.

In this paper, a new optimization problem for power control of DSSS systems is formulated first, and it is based on the joint consideration of the Tx transmission power and link quality in a global objective functional form as follows:

$$
L(\mathbf{p})=\left(\sum_{i=1}^{N} p_{i}\right)^{-1}+\tilde{\lambda}_{0} \sum_{i=1}^{N} \operatorname{SINR}_{i}(\mathbf{p})
$$

We consider this form of utility function in (1) due to its simplicity, and yet it takes into account both link quality and distortion. Similar utility function has been adopted in [7]. 
Although orthogonal spreading codes, e.g. Walsh code, can totally eliminate cross-correlation, generally they have high partial cross-correlation peaks which can cause a high false lock rate during capture in an asynchronous system. The problem formulated and studied in [6], which does not take into account spreading effects, can not be applied to a DSMA system where non-orthogonal scrambling codes are used. Msequences can have a very low peak out-of-phase autocorrelation and a bounded peak cross-correlation, but the number of codes are very limited and this renders it only suitable for a small network allowing very few simultaneous communicating pairs [10], [5]. Non-orthogonal codes including the Gold code [12], small set and large set of the Kasami code [11] are more suitable for waveform scrambling in a DSSS system with large set of codes. Peak out-of-phase autocorrelation and cross-correlation [4], [5] with these codes is non-zero and must be taken into account in power allocation. Here, only the peak correlations of spreading codes are of interest since asynchronous transmission (which is commonly used) is assumed in this paper. As an example, for $n$ not of multiple of 4 , the Gold code can generate $2^{n}+1$ spreading codes of period $2^{n}-1$ with a three-valued partial cross-correlation and autocorrelation,

$$
-1,-2^{\left\lfloor\frac{n+2}{2}\right\rfloor}-1,2^{2^{\left\lfloor\frac{n+2}{2}\right\rfloor}}-1,
$$

out of

$$
\frac{\phi\left(2^{n}-1\right)}{n}
$$

$\mathrm{m}$-sequences where $\phi$ is the Euler Totient function.

In general, cross-interference and self-interferences should be taken into account when considering power allocation in a DSSS wireless network. However, we only consider the cross interference of the direct path, the results can be extended to include these multi-path components of cross-interferences. The proposed scheme can be applied to ad hoc networks (where DSSS radios are employed) or uplink carrier DSSS networks.

This paper is organized as follows: Section II presents the model and problem formulation. Section III presents the iterative algorithm. Section IV presents the numerical and simulation results and Section V concludes this paper.

\section{Power Optimization Problem Formulation}

We first introduce notation to be used for the model equations in this section. Let $I_{c}^{k}$ and $I_{a}^{k}$ be the interferences of the $\mathrm{k}$-th $\mathrm{Rx}$ radio due to cross-correlation from other cochannel users and autocorrelation of $k$ multipath components, respectively. For $1 \leq t \leq T$, let $\hat{C}_{t}^{i}$ be the t-th bit (-1 or 1 ) of the spread code $s_{i}$, then define,

$$
\begin{aligned}
& \hat{\theta}_{i k}(0, t)=\frac{1}{T} \sum_{v=1}^{t} \hat{C}_{v}^{k} \hat{C}^{i}(v-t) \bmod T \\
& \hat{\theta}_{i k}(t, T)=\frac{1}{T} \sum_{v=t}^{T} \hat{C}_{v}^{k} \hat{C}_{v-t}^{i}
\end{aligned}
$$

By noticing the polarity differences of different spreading codes to the intended receiver, the peak cross-correlation function [5] is defined as follows:

$$
\begin{array}{r}
\theta\left(s_{i}, s_{k}\right)=\max _{t}\left\{\hat{\theta}_{i k}(1, t)+\hat{\theta}_{i k}(t+1, T),\right. \\
\left.\left|\hat{\theta}_{i k}(1, t)-\hat{\theta}_{i k}(t+1, T)\right|\right\}
\end{array}
$$

Let the k-th cross-correlation auxiliary vector $\omega^{k}=$ $\left(\theta\left(s_{1}, s_{k}\right), \theta\left(s_{2}, s_{k}\right), \cdots, \theta\left(s_{N}, s_{k}\right)\right)^{t r}$, the SINR vector $l(p)$ of these $N$ users is a function of $\mathbf{p}$. Let $\Delta$ be the unit chip delay operator and $\Delta^{t}$ for a t-chip delay operator on a code, let $\tau_{k}$ be the multi-path delay on the k-th path. The SINR at the k-th receiver can be estimated by (5), assuming that only the SINR on the RF signal is considered:

$$
\begin{aligned}
\mathbf{l}(\mathbf{p})[k] & =\frac{p_{k} g_{k k}}{I_{c}^{k}+p_{k} g_{k k} I_{a}^{k}+\sigma^{2}} \\
I_{c}^{k} & =<\mathbf{p} \otimes \boldsymbol{\omega}^{k}, \mathbf{G}_{\mathbf{k}}>_{N, k} \\
I_{a}^{k} & =<\Delta^{\tau_{k}} \mathbf{s}, \mathbf{s}>_{k}
\end{aligned}
$$

This SINR formula takes into account both the autocorrelation and cross-correlation as compared to previous formulae, e.g. [6]. A vector of SINR from these $N$ co-channel users is denoted by $l(\mathbf{p})$ whose $\mathrm{k}$-th term is given by (5).

From (1) above, our power/quality optimization problem (PQOP) is formulated as follows:

$$
\mathbf{p}^{*}=\underset{\overline{\mathbf{p}} \leq \mathbf{p} \leq \overline{\mathbf{P}}, \overline{\mathbf{l}} \leq l(\mathbf{p})}{\operatorname{argmax}}\left(\frac{1}{\|\mathbf{p}\|_{1}}+\|\boldsymbol{\lambda} \otimes \mathbf{l}(\mathbf{p})\|_{1}\right)
$$

where, $\overline{\mathbf{p}}$ and $\overline{\mathbf{P}}$ are the lower and upper bounds of power, respectively, and $\overline{\mathbf{l}}$ is the lower bound of the SINR vector. The lower bound of SINR vector $\overline{1}$ is set based on application requirements, e.g. desired channel bit error rate (BER) and/or frame error rate (FER), while the lower bound of power vector is set based on collision link quality indication (LQI) threshold. The upper bound on power is due to practical considerations e.g., ITU or FCC regulation on radio emission power [3]. Note that the above problem reduces to a pure power allocation problem when $\boldsymbol{\lambda}=0$ and it reduces to a link quality optimization problem when for all $\lambda_{i}$ we have $\lambda_{i} \gg 1$.

Note that this formulation is independent of whether the despreading and symbol decision statistics computation are done in analog domain or digital domain (i.e. oversampling of $\mathrm{RF}$ signal at a rate greater than the chip rate). Let

$$
\lambda_{i}=\tilde{\lambda}_{0}(1 \leq i \leq N)
$$

then (1) is a special case of (6). Although other norms (e.g. $\left.L^{2}, L^{\infty}\right)$ of (6) are possible, we use the $L^{1}$ norm in this paper.

\section{Proposed Iterative Distributed Power ALLOCATION SCHEME}

Without loss of generality, in this section and Appendix A and $\mathrm{B}$, we consider $\mathrm{j}$-th radio with power $p_{j}$ and spread code $s_{j}$. Let $\eta=\max _{i}\left\{\theta\left(s_{i}, s_{j}\right)\right\}$ and $\beta=\max _{i}\left\{\left\langle\Delta^{\tau_{i}} s_{j}, s_{j}\right\rangle\right\}$, 
In order to formulate a distributed problem, each radio tries to maximize the following individual objective functional:

$$
L\left(p_{j}\right)=\frac{1}{p_{j}}+\frac{\lambda_{j} g_{j j} p_{j}}{C_{j}+\beta g_{j j} p_{j}+\sigma^{2}}
$$

where,

$$
C_{j}=\eta \sum_{i=1, i \neq j}^{N} p_{i} g_{j i}
$$

the sum in (8) is over the set of simultaneously communicating pairs except the pair in question with the $\mathrm{j}$-th user using power $p_{j} ; \lambda_{j}$ is the functional coefficient for this radio.

Let $x_{j}$ be the minimal SINR (predetermined threshold) of the $\mathrm{Rx}$ to which the Tx radio of the $\mathrm{j}$-th user is communicating. We assume that the power $p_{j}$ is normalized, i.e., $p_{j} \in(0,1]$, and the SINR condition for this radio is as follows:

$$
x_{j} \geq \frac{p_{j} g_{j j}}{C_{j}+\beta g_{j j} p_{j}+\sigma^{2}}
$$

Remark 1: When cross-interference is taken as fixed, for two extreme cases of $\lambda_{j} \gg 1$ and $\lambda_{j} \ll 1$, the optimal solutions at a single radio is trivial.

For $\lambda_{j} \gg 1$, the local optimal power is the biggest possible power which equals 1 . This is due to that transmission power is the dominating factor on the local objective functional. For $\lambda_{j} \ll 1$, the local optimal power is the smallest power possible which is given by (10) (obtained by applying the SINR constraint):

$$
p_{j}^{*}=\frac{x_{j}\left(C_{j}+\sigma^{2}\right)}{g_{j j}\left(1-x_{j} \beta\right)}
$$

This is due to that SINR is the dominating factor on the local objective functional.

For other cases, Theorem 1 and Theorem 2 stated below give the optimal power allocation. We first state and prove the following propositions.

Proposition 1: For the individual objective functional given in (7), the maximum Tx power can be either 1 or $p_{j}^{*}$.

Proof: The proof is given by examining the root $p_{j}^{0}$ of the first derivative of $L\left(p_{j}\right)$, then evaluating the second derivative at $p_{j}^{0}, L^{\prime \prime}\left(p_{j}^{0}\right)$, which turns out to be greater than 0 . The first derivative of $L$ is given in (11).

$$
L^{\prime}=\frac{\lambda_{j}\left(C_{j}+\sigma^{2}\right)}{\beta^{2} g_{j j}} \frac{1}{\left(p_{j}+\tilde{U}\right)^{2}}-\frac{1}{p_{j}^{2}}
$$

where, $\tilde{U}=\frac{C_{j}+\sigma^{2}}{\beta g_{j j}}>0$ and $\beta g_{j j}>0$. This root of the first derivative of $L$ is given in (12) note that $\lambda_{j}>\beta^{2} g_{j j} /\left(C_{j}+\delta^{2}\right)$ always holds in practice.

$$
p_{j}^{0}=\frac{C_{j}+\sigma^{2}}{\sqrt{\lambda_{j} g_{j j}\left(C_{j}+\sigma^{2}\right)}-\beta g_{j j}}
$$

and

$$
L^{\prime \prime}\left(p_{j}^{0}\right)=\frac{2}{\tilde{K}}\left(\sqrt{\lambda_{j} g_{j j}\left(C_{j}+\sigma^{2}\right)}-\beta g_{j j}\right)^{4}
$$

where,

$$
\tilde{K}=\left(C_{j}+\sigma^{2}\right)^{3.5} \sqrt{\lambda_{j} g_{j j}}
$$

is an expression which is always positive even so when only one radio is active (i.e. $C_{j}=0$ ). Therefore, $L^{\prime \prime}\left(p_{j}^{0}\right)$ in (13) is always greater than 0 . When $\lambda_{j}=\frac{\beta^{2} g_{j j}}{C_{j}+\sigma^{2}}$, let $p_{j}^{*}=p_{j}^{0}, L^{\prime}$ can be rewritten as (14).

$$
L^{\prime}=\frac{1}{\left(p_{j}+\tilde{U}\right)^{2}}-\frac{1}{p_{j}^{2}}<0 \quad \forall p_{j} \in(0,1]
$$

Therefore, the maximum of the objective functional is obtained when $\lambda_{j}$ satisfies (15).

$$
\lambda_{j} \leq \frac{\beta^{2} g_{j j}}{C_{j}+\sigma^{2}}
$$

For other cases, the maximum power at 1 yields the maximal utility.

Proposition 2: When the SINR satisfies:

$$
x_{j}=\frac{g_{j j}}{C_{j}+\sigma^{2}+\beta g_{j j}}
$$

then $L(1)=L\left(p_{j}^{*}\right)$.

Proof: By noticing the following formulae:

$$
\begin{gathered}
L(1)=1+\frac{\lambda_{j} g_{j j}}{C_{j}+\sigma^{2}+\beta g_{j j}} \\
L\left(p_{j}^{*}\right)=\lambda_{j} x_{j}+\frac{g_{j j}\left(1-\beta x_{j}\right)}{x_{j}\left(C_{j}+\sigma^{2}\right)}
\end{gathered}
$$

when the condition of the proposition holds, the following equations are valid:

$$
\begin{aligned}
\lambda_{j} x_{j} & =\frac{\lambda_{j} g_{j j}}{C_{j}+\sigma^{2}+\beta g_{j j}} \\
1 & =\frac{g_{j j}\left(1-\beta x_{j}\right)}{x_{j}\left(C_{j}+\sigma^{2}\right)}
\end{aligned}
$$

By (17) and (18) above, this proposition follows.

Theorem 1: We distinguish two cases: (a)

$$
\lambda_{j}<\frac{\left(C_{j}+\sigma^{2}+\beta g_{j j}\right)^{2}}{g_{j j}\left(C_{j}+\sigma^{2}\right)}
$$

with

$$
x_{j} \leq \frac{g_{j j}}{C_{j}+\sigma^{2}+\beta g_{j j}}
$$

and (b)

$$
\lambda_{j}>\frac{\left(C_{j}+\sigma^{2}+\beta g_{j j}\right)^{2}}{g_{j j}\left(C_{j}+\sigma^{2}\right)}
$$

with

$$
x_{j}>\frac{C_{j}+\sigma^{2}+\beta g_{j j}}{\lambda_{j}\left(C_{j}+\sigma^{2}\right)}
$$

For (a) or (b) the local optimal power allocation is $p_{j}^{*}$.

Proof: in Appendix A.

Note that these two cases are not exhaustive, but nevertheless, they are the most interesting cases. For the other borderline cases, definitive answers are hard to obtain.

By Theorem 1, we know that the local optimal power is the lowest link SINR satisfying power for the given cases. For all other cases, local optimal may be $p_{j}^{*}$ or 1 (See Proposition 1 ). The next theorem states that the local optimal for those two 
cases in Theorem 1 is actually the best that can be obtained in terms of the global optimal.

Theorem 2: If the $\mathrm{j}$-th link has local optimal at $p_{j}^{*}$ which is the lowest SINR satisfying power of the link, then the global optimal power allocation w.r.t. this link is also $p_{j}^{*}$.

Proof: in Appendix B.

A new distributed power allocation scheme for radios is proposed based on Theorem 2 and Theorem 1. The interference can be estimated by the received signal strength indicator (RSSI) from the radio antenna. RSSI is a quantity indicating the signal strength the radio can compute locally [1]. This algorithm executes in a distributed iterative manner as follows where the superscript denotes the iteration number and $\left(\sigma^{(k)}\right)^{2}$ is the noise density observed during the k-th iteration.

Algorithm 1: Each node $j$ iterates, using as inputs the vectors $\mathbf{s}$ (spreading code vector of active radios) and $\mathbf{p}$ (prior power allocation vector), by Theorem 1 (for local optimization computation) and Theorem 2 (for global optimization), local computation can yield optimal objective functional to obtain $p_{j}^{*}$ as follows:

0. $p_{j} \leftarrow p_{j}^{0}$ (lowest power level)

- iteration (1.) - (3.), for the k-th iteration

1. $p_{j} \leftarrow p_{j}^{(k+1)}$ (next available power level)

2. $C_{j}^{(k+1)} \leftarrow \operatorname{RSSI}^{(k)}-\beta g_{j j} p_{j}-\left(\sigma^{(k)}\right)^{2}$

3. if

$$
\left|L\left(p_{j}^{k}\right)-L\left(p_{j}^{(k+1)}\right)\right|<\text { tolerance }
$$

select $p_{j}^{*}=p_{j}^{(k+1)}$ and exit the iteration process.

$$
\text { - else, } k \leftarrow(k+1) \text { and goto step } 1 \text {. }
$$

Step 2. is to estimate the aggregated interference with a given local power assignment. Step 3. is to select a power level locally which sujects to the given tolerance on the changes of local functional. Note that there is no need to synchronize these active radios and new power level from other active radios can be incorporated into the computation of an iteration once this information along with the spreading code assignment is made available to the local radio or estimated using RSSI by the local radio.

The algorithm is performed by each active node in a distributed manner. However, node $j$ requires information from the other nodes at each iteration to compute $C_{j}^{(k+1)}$, and this information is obtained through $\operatorname{RSSI}^{(k)}$ during the $\mathrm{k}$-th iteration from the antenna.

By Theorem 1, and the convexity of the local functional around $p_{j}^{*}$ (note that the local function is not a convex function for certain combination of multiplier $\lambda_{j}$ and RSSI), the convergence of Algorithm 1 is certain since only powers close to these two extreme cases are interesting and examined. The salient feature of Algorithm 1 is its local computation performed in a distributed manner and the guarantee of global optimum following Theorem 2 .

\section{Numerical AnAlysis And Simulation Study}

We first perform numerical analysis using MATLAB on the local optimization problem in which we use a two-radio model to show some analytic results and we also use a multi-radio model to show some analytic local optimization results. Then results on simulation using the configuration of CC2420 ( [1]) are presented focusing on energy dissipation and bit error rate. Our study uses SINR estimation of uncoded DSSS system and BER bounds on uncoded DSSS systems as shown in [8].

Figure 1(a) - Figure 1(c) (for small file sizes, only the lower quadrant is plotted) show the feasible spaces of power allocation optimization for three different adjusting factors of the local functional. These figures provide the intuition of determining the optimal power allocation using Theorem 1 and Theorem 2. Based on the shape of the functional, the minimizing powers can only be the global minimal power if it falls into the feasible region; otherwise, the minimizing power can only the minimal power along the lower edge of the region.

Figure 2 shows how cross interference affects the local optimal and how RSSI affects the optimization in different regimes of $\lambda$ where only curves using the feasible regions are plotted. These plots are obtained from the local objective functional. From Fig. 2, in large $\lambda$ regime, optimal power allocation can shift between the normalized maximal power 1 and $\tilde{p}$. In the low $\lambda$ regime, the optimal power allocation is consistently at $\tilde{p}$ which makes effective power allocation possible.

In what follows, an initial implementation of the proposed power control scheme is given using the QualNet simulator [2], and simulation is performed with three different network configuration scenarios. The simulation parameters are given in Table I. The nodes are deployed randomly and we tested three scenarios with different numbers of nodes involved. Figure 3 shows the energy consumption for power control using the proposed scheme in a multi-hop network, distance based power control, SINR based power control and a fixed power allocation scheme. Each group has four numbers corresponding to these schemes where only the best static allocation scheme is plotted. Only under the proposed scheme, the energy savings are high while the BER is kept relatively low. The energy reduction compared to the best fixed power scheme is close to $25 \%$. The corresponding bit error rates are shown in Fig. 4.

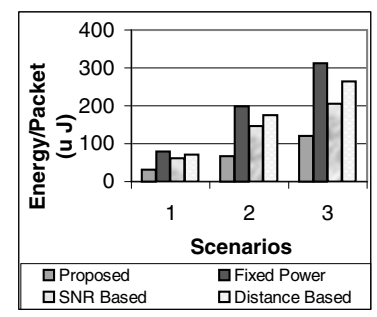

Fig. 3. Energy Dissipation Comparison

\section{Conclunsions}

In this paper, a novel scheme on power allocation for direct-sequence spread spectrum systems is proposed. Power is allocated to optimize a local objective functional and this 


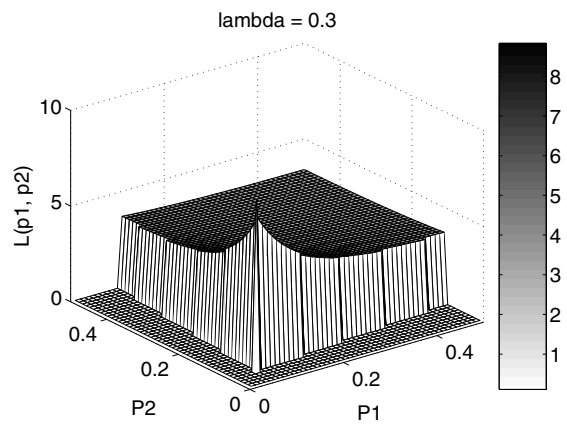

(a) $\lambda=0.3$

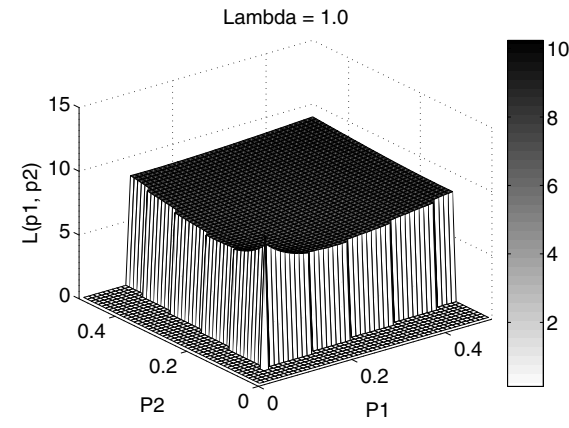

(b) $\lambda=1.0$

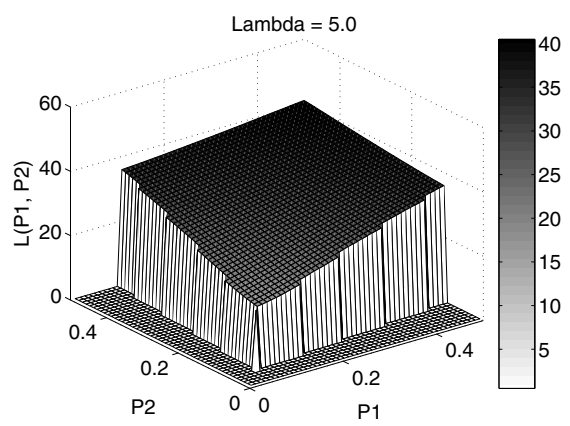

(c) $\lambda=5.0$

Fig. 1. Analytic Results on the Two-radio Case

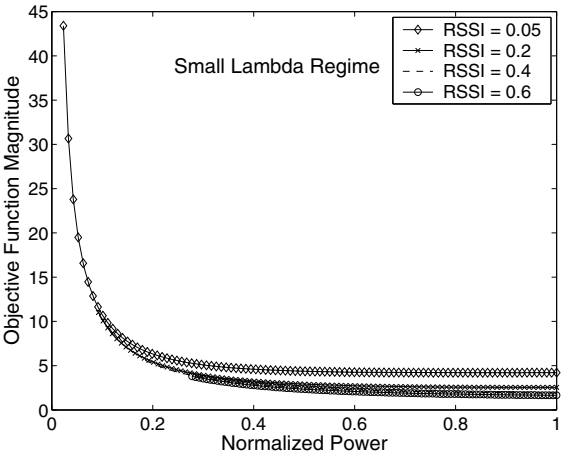

(a) Low $\lambda$ Regime

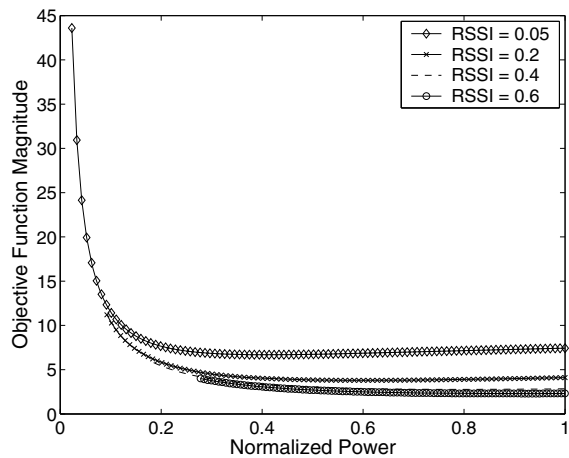

(b) $\lambda=1.0$

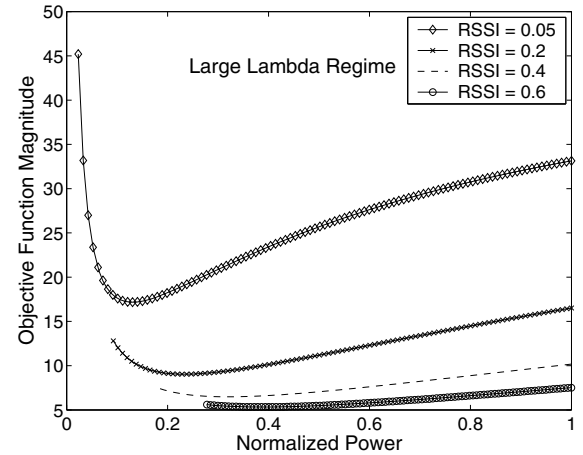

(c) High $\lambda$ Regime

Fig. 2. Cross Interference and Local Optima

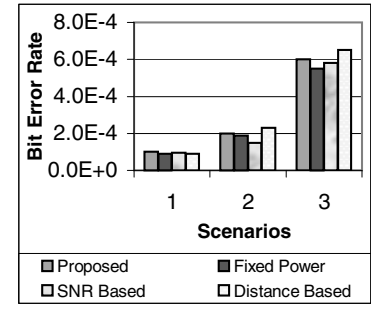

Fig. 4. Bit Error Rate Comparison

scheme can guarantee a global optimum. Numerical analysis and simulation results show that significant energy savings are obtained compared to the case without power control.

\section{REFERENCES}

[1] Chipcon AS, "CC2420 Radio Model [online]," Available: http://www.chipcon.com.

[2] Scalable Network Technologies, QualNet 3.7 [online]. Available: http://www.scalable-networks.com.

[3] U.S. Federal Communications Commission, "FCC Part 15.247 - Spread Spectrum Radio Systems [online]," Available: http://www.fcc.gov.
[4] M. B. Pursley and D. V. Sarwate, "Performance Evaluation for PhaseCoded Spread-Spectrum Multiple-Access Communication - Part II: Code Sequence Analysis," IEEE Transactions on Communications, COM25(8): $800-803$, August, 1977.

[5] D. V. Sarwate and M. B. Pursley, "Crosscorrelation Properties of Pseudorandom and Related Sequences," Proceedings of The IEEE, 68(5): $593-619$, May, 1980.

[6] N. Bambos, "Toward Power-Sensitive Network Architectures in Wireless Communications: Concepts, Issues, and Design Aspects," IEEE Personal Communications, June, 1998.

[7] B. S. Krongold, K. Ramchandran, and D. L. Jones, "Computationally Efficient Optimal Power Allocation Algorithms for Multicarrier Communication Systems," IEEE Transactions on Communications, 48(1): 23 - 27, Jan., 2000.

[8] M. B. Pursley, "Performance Evaluation for Phase-Coded SpreadSpectrum Multiple-Access Communication - Part I: System Analysis," IEEE Transactions on Communications, COM-25(8): 795 - 799, August, 1977.

[9] Y.-H. Lin and R. L. Cruz, "Power Control and Scheduling for Interfering Links," Proc. of IEEE Information Theory Workshop, Oct. 2004.

[10] J. H. Lindholm, "An Analysis of the Pseudo-Randomness Properties of Subsequences of Long m-Sequences," IEEE Transactions on Information Theory, IT-14(4): 569 - 576, July, 1968.

[11] T. Kasami, "The weight enumerators for several classes of subcodes of the 2nd-order binary Reed-Muller Codes," Information Control, 18(4): 369 - 394, May, 1971.

[12] R. Gold, "Optimal binary sequences for spread spectrum multiplexing," 
TABLE I

SiMUlation PARAMETERS

\begin{tabular}{|c|c|}
\hline Channel Frequency & $2.4 \mathrm{GHz}$ \\
\hline Data Rate & $250 \mathrm{bits} / \mathrm{s}$ \\
\hline Radio Type & Accumulating Noise \\
\hline Fading Model & Ricean \\
\hline Propagation Pathloss Model & Two-Ray Ground \& Reflection \\
\hline Maximum Tx Power & $15 \mathrm{dBm}$ \\
\hline Number of Power Levels & $-91.0 \mathrm{dBm}$ \\
\hline Radio Rx Sensitivity & $10.0 \mathrm{dBm}$ \\
\hline Radio Rx SINR Threshold & $-94.0 \mathrm{dBm}$ \\
\hline AWGN Noise Power & $8,12,21$ \\
\hline Number of Simultaneous Links & 5 \\
\hline Number of Multi-path & 1.0 (LOS) 0.70 .20 .10 .05 \\
\hline Normalized Multi-path Factors & 03101112 \\
\hline Multi-path Phase Offset (chips) & $-13 \mathrm{~dB}($ estimate) \\
\hline Ricean K Factor & Period 127 Gold Code \\
\hline Spreading Code & 1 (15), 2 (35), 3 (75) \\
\hline Scenarios (\# of nodes) &
\end{tabular}

IEEE Transactions on Information Theory, IT-13: 619 - 621, October, 1968.

[13] J. Jubin and J. Tornow, "The darpa packet radio network protocols," Proceedings of the IEEE, 75(1):21 - 32, January 1987.

\section{ApPENDiX A: Proof of TheOREM 1}

Proof: Let us introduce a variable $t$ to the right of (16) in Proposition 2, that is

$$
x_{j}=\frac{t g_{j j}}{C_{j}+\sigma^{2}+\beta g_{j j}}
$$

where $t>0$, and let us introduce two functions, $f_{1}\left(x_{j}, \lambda_{j}\right)$ and $f_{2}\left(x_{j}\right)$ as follows:

$$
\begin{aligned}
f_{1}\left(x_{j}, \lambda_{j}\right) & =\lambda_{j} x_{j}-\frac{\lambda_{j} g_{j j}}{C_{j}+\sigma^{2}+\beta g_{j j}} \\
f_{2}\left(x_{j}\right) & =1-\frac{g_{j j}\left(1-x_{j} \beta\right)}{x_{j}\left(C_{j}+\sigma^{2}\right)}
\end{aligned}
$$

$f_{1}\left(x_{j}, \lambda_{j}\right)$ and $f_{2}\left(x_{j}\right)$ are both strictly increasing on $x_{j}$, and they are either both greater than 0 or both less than 0 for $\lambda_{j}>0$ as seen by factoring out $\lambda_{j} x_{j}$ from $f_{1}\left(x_{j}, \lambda_{j}\right)$. After some simplification and (21), we have

$$
\begin{aligned}
f_{1}\left(t, \lambda_{j}\right) & =\frac{\lambda_{j} g_{j j}(t-1)}{C_{j}+\sigma^{2}+\beta g_{j j}} \\
f_{2}(t) & =\frac{(t-1)\left(C_{j}+\sigma^{2}+\beta g_{j j}\right)}{t\left(C_{j}+\sigma^{2}\right)}
\end{aligned}
$$

Considering the function in (22)

$$
W\left(t, \lambda_{j}\right)=\frac{f_{1}\left(t, \lambda_{j}\right)}{f_{2}(t)} \text { for } t \neq 1 .
$$

$W\left(t, \lambda_{j}\right)$ determines the relationship of $L\left(p_{j}^{*}\right)$ and $L(1)$, and when $t \neq 1$, it can be rewritten as follows:

$$
W\left(t, \lambda_{j}\right)=\frac{g_{j j}\left(C_{j}+\sigma^{2}\right) \lambda_{j} t}{\left(C_{j}+\sigma^{2}+\beta g_{j j}\right)^{2}}
$$

and when $t=1, W\left(t, \lambda_{j}\right)$ can be defined as $\lim _{t \rightarrow 1} W\left(t, \lambda_{j}\right)$; however this is of no interest here. For

$$
\lambda_{j}<\frac{\left(C_{j}+\sigma^{2}+\beta g_{j j}\right)^{2}}{g_{j j}\left(C_{j}+\sigma^{2}\right)}
$$

$L\left(p_{j}^{*}\right)>L(1)$ when $t>1$. For

$$
\lambda_{j}>\frac{\left(C_{j}+\sigma^{2}+\beta g_{j j}\right)^{2}}{g_{j j}\left(C_{j}+\sigma^{2}\right)}
$$

$L\left(p_{j}^{*}\right)>L(1)$ when $t<1$. By Proposition 1, this theorem follows.

\section{ApPendiX B: Proof of TheOREM 2}

Proof: For $N$ simultaneous communicating links, denote the global power allocation $\mathbf{p}^{*}$ as follows:

$$
\mathbf{p}^{*}=\left(p_{1}, p_{2}, \cdots, p_{N}\right)
$$

If the $\mathrm{k}$-th link satisfies the condition in the theorem, and $p_{k} \neq$ $p_{k}^{*}$, a new power allocation $\mathbf{p}^{\prime}$ can be constructed as follows:

$$
\mathbf{p}^{\prime}=\left(p_{1}, \cdots, p_{k}^{*}, \cdots, p_{N}\right)
$$

To show that $\mathbf{p}^{\prime}$ yields a lower value to the global objective functional, there are two cases to consider: (1) $p_{k}<p_{k}^{*}$ and (2) $p_{k}>p_{k}^{*}$. These two cases could happen because $\lambda_{k}$ could vary from 0 to $\infty$. We next consider the case $p_{k}>p_{k}^{*}$ since this is a more practical case, and a similar argument to the one given below can be conducted on the other case. When $p_{k}>p_{k}^{*}, \mathbf{p}^{\prime}$ will yield a lower value to the global objective functional. This is true because the following two claims are valid:

1. $\mathbf{p}^{\prime}$ still satisfies all links' SINR requirements, i.e., it falls into the feasible region.

2. $L\left(\mathbf{p}^{*}\right)<L\left(\mathbf{p}^{\prime}\right)$.

For Item (2.), expanding the terms,

$$
\begin{aligned}
L\left(\mathbf{p}^{\prime}\right)-L\left(\mathbf{p}^{*}\right)= & \frac{\lambda_{k} g_{j k} p_{k}^{*}}{C_{j}+\beta g_{j k} p_{k}^{*}+\sigma^{2}}+\left\|\mathbf{p}^{\prime}\right\|^{-1}- \\
& \frac{\lambda_{k} g_{j k} p_{k}}{C_{j}+\beta g_{j k} p_{k}+\sigma^{2}}-\left\|\mathbf{p}^{*}\right\|^{-1}+U_{j}
\end{aligned}
$$

where,

$$
\begin{aligned}
U_{j}= & \left\|\boldsymbol{\lambda} \otimes \mathbf{l}\left(\mathbf{p}^{\prime}\right)\right\|-\left\|\boldsymbol{\lambda} \otimes \mathbf{l}\left(\mathbf{p}^{*}\right)\right\|+ \\
& \frac{\lambda_{k} g_{j k} p_{k}^{*}}{C_{j}+\beta g_{j k} p_{k}^{*}+\sigma^{2}}- \\
& \frac{\lambda_{k} g_{j k} p_{k}}{C_{j}+\beta g_{j k} p_{k}+\sigma^{2}}
\end{aligned}
$$

Note that

$$
U_{j}>0
$$

Equation (23) is due to the fact that $p_{k}^{*}$ is the optimal power for the $\mathrm{k}$-th radio and $\mathbf{p}^{*}$ is the optimal power vector (note that $\mathcal{L}^{1}$ norm is used). By noticing that

$$
\frac{1}{p_{k}}+\frac{\lambda_{k} g_{k k} p_{k}}{C_{j}+\beta g_{k k} p_{k}+\sigma^{2}}<\frac{1}{p_{k}^{*}}+\frac{\lambda_{k} g_{k k} p_{k}^{*}}{C_{j}+\beta g_{k k} p_{k}^{*}+\sigma^{2}}
$$

then the strict inequality of Item (2.) holds true. Therefore, a better power allocation scheme is obtained, and this is a contradiction. 